\title{
CARTA AL EDITOR \\ Virtualidad en el curso teórico de Medicina Interna en estudiantes de V, VI y VII semestre a propósito de la pandemia COVID-19 durante el primer semestre del 2020, Facultad de Medicina, Escuela de Ciencias de la Salud, Universidad Pontificia Bolivariana, Medellín, Colombia: divulgación de un ejercicio académico
}

Fecha de recibido: 23 de mayo de 2020 Fecha de aprobación: 30 de junio de 2020

Forma de citar este artículo: Luján-Piedrahita M. Experiencia de virtualidad en el curso teórico de Medicina Interna en estudiantes de $\mathrm{V}, \mathrm{VI}$ y VII semestre a raíz de la pandemia COVID-19 durante el primer semestre del 2020, Facultad de Medicina, Escuela de Ciencias de la Salud, Universidad Pontificia Bolivariana, Medellín, Colombia: divulgación de un ejercicio académico. Med UPB. 2020;39(2):66-72 DOI:10.18566/medupb.v39n2.a11

1 Coordinación de Área Clínicas del Adulto (Pregrado), Facultad de Medicina, Escuela Ciencias de la Salud, Universidad Pontificia Bolivariana. Medellín, Colombia.

2 Clínica de Oncología Astorga. Medellín, Colombia.

Dirección de

correspondencia: Mauricio Luján-Piedrahita. Correo electrónico: mlujan@une. net.co
Virtuality in the internal medicine theoretical course in students of V, VI and VII semester as a result of the COVID-19 pandemic during the first semester of 2020, School of Medicine, School of Health Sciences, Universidad Pontificia Bolivariana, Medellín, Colombia: Disclosure of an academic exercise / Virtualidade no curso teórico de Medicina Interna em estudantes de V, VI e VII semestre a raiz da pandemia COVID-19 durante o primeiro semestre de 2020, Faculdade de Medicina, Escola de Ciências da Saúde, Universidad Pontificia Bolivariana, Medellín, Colômbia: divulgação de um exercício acadêmico

Mauricio Luján-Piedrahíta ${ }^{1,2}$

Señor Editor:

Estos son tiempos sin precedentes modernos, la aparición del coronavirus 19 (COVID-19) y sus consecuencias han interrumpido y afectado la educación médica. Aunque la prioridad ha sido y debe ser la atención a los pacientes y las comunidades, esta pandemia implica desafíos logísticos y preocupaciones sobre la formación de los futuros médicos, por lo que se requiere una atención intensa y rápida por parte de las escuelas de medicina, directivos (universitarios y hospitalarios) y educadores médicos ${ }^{1}$.

Durante la última década, la mayoría de las facultades de medicina están transformando la pedagogía, mediante la eliminación o reducción de actividades teóricas y la utilización de tecnologías y laboratorios de simulación para sustituir y mejorar procesos, tanto de ciencias básicas como clínicas. Así mismo, están actualizando los modelos de aprendizaje que se facilitan por equipos interprofesionales y transdisciplinarios.

Se ha reducido el programa de ciencias básicas y se ha adelantado el inicio de la formación clínica, mientras se integran conocimientos básicos según avanza la práctica. Hoy en día, en la mayoría de las facultades, los estudiantes solo se reúnen durante los primeros semestres para resolver problemas interactivos o debatir en grupos, mientras inician tempranamente su inmersión en entornos asistenciales. Generalmente, los últimos semestres pueden ser individualizados, con estudiantes que participan en rotaciones clínicas avanzadas, internados especiales y proyectos de investigación ${ }^{2,3}$.

La práctica clínica, el contacto con pacientes y el ambiente sanitario es fundamental en la formación médica. Ante esta pandemia en la que el distanciamiento social es la 
única estrategia preventiva eficaz, hasta que se desarrolle una vacuna, un tratamiento o ambos (por ahora no cercanos), se hace imperativo, no solo como estrategia ante el incierto panorama, sino como alternativa complementaria a las actividades formativas futuras o ante nuevos brotes o eventos postpandemia, la creación de alternativas de aprendizaje en grupos pequeños. Los ámbitos de interacción incluyen sesiones de laboratorio, simulación, tecnología (telemedicina) y auténticos entornos de atención al paciente para la instrucción clínica ${ }^{4}$.

A raíz de la pandemia, en marzo de 2020, la recomendación, mundial y local fue que las facultades de medicina diseñaran estrategias de acompañamiento durante la pausa en las actividades presenciales y clínicas de los estudiantes, sugerencia acatada por la Facultad de Medicina de la Universidad Pontificia Bolivariana (UPB). Las facultades médicas de casi todo el mundo han hecho una rápida transición del plan de estudios y evaluaciones de pregrado a formatos en línea. Algunas escuelas han diseñado formatos de pequeños equipos virtuales, con algunas sesiones de habilidades clínicas en línea. Las actividades virtuales parecen ser funcionales, pero los resultados de estos cambios requieren evaluación ${ }^{1,4}$.

\section{Encuesta a estudiantes de pregrado}

Como parte del análisis habitual al final del curso y con el objetivo de evaluar la experiencia y percepción de los estudiantes sobre la implementación súbita y total de la virtualidad en la parte teórica de los cursos de Medicina Interna I, II y III, se aplicó una encuesta virtual, voluntaria y anónima a los estudiantes (niveles V, VI y VII) durante el primer semestre del año 2020.

\section{Resultados de la encuesta}

Participaron 130 estudiantes ( 51 de V nivel, 37 de VI nivel y 42 de VII nivel), 74.6\% sexo femenino. El 65\% manifestó haber tenido experiencia previa con actividades académicas virtuales. El 18\% manifestó tener dificultades para acceder a Internet. Un 16\% consideró que habría sido mejor cancelar el semestre. E1 65\% de los estudiantes prefiere la formación presencial, el 59\% considera que aprende menos en una sesión virtual, percepción que varía con el nivel (70\% en V, 62\% en VI y 42\% en VII).

Dentro de las ventajas que encuentran en las actividades virtuales (Figura 1), más del 70\% considera importante el acceso al contenido durante las 24 horas del día, lo que permite adaptar el estudio a la disponibilidad de tiempo y lugar, con solo conexión a Internet.Más del 60\% incluye el ahorro de tiempo y dinero y mayor oportunidad para analizar la información. La principal desventaja está relacionada con la Red (Figura 2), el 91\% considera que una mala conexión, fallas en equipos o material generan retrasos e interrupciones. E1 71\% de los encuestados creen que sin una rutina programada de estudios se pueden descontrolar los horarios para otras actividades. Más del $60 \%$ piensan que se crea una brecha en la relación alumno-maestro al perder contacto personal.

E1 84\% (Figura 3) considera que la enseñanza presencial es más didáctica y práctica, al 72\% le genera mayor interés, disposición y motivación, y el $68 \%$ considera que permite la aplicación de técnicas específicas al tener el profesor más conocimiento del grupo. Un 66\% está de acuerdo con que la formación presencial es más eficiente, al no depender del funcionamiento de herramientas de audio o video. Más del 55\% de los estudiantes creen que la presencialidad refuerza valores éticos y aporta más al

Figura 1. Principales ventajas que los estudiantes de pregrado de Medicina Interna I, II y III encuentran en las actividades virtuales.
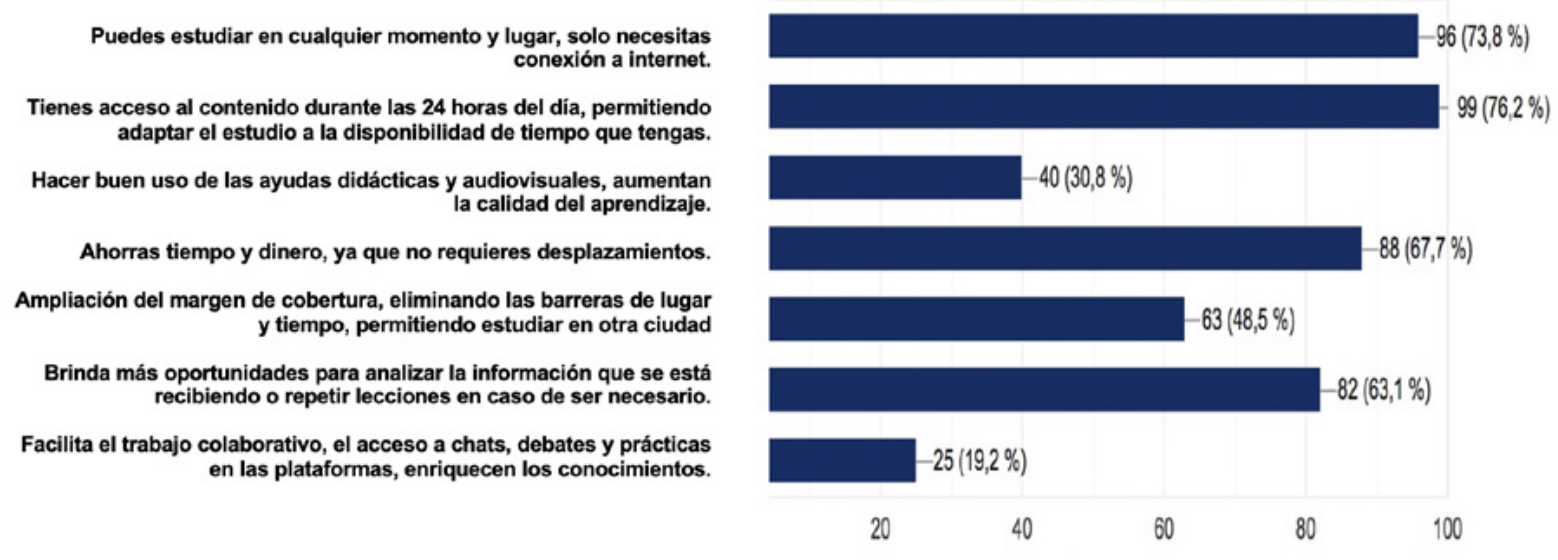
Figura 2. Principales desventajas que los estudiantes de pregrado de Medicina Interna I, II y III encuentran en las actividades virtuales.

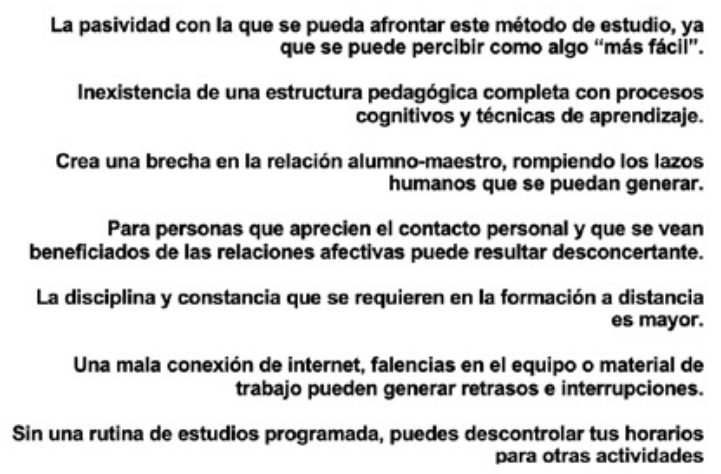

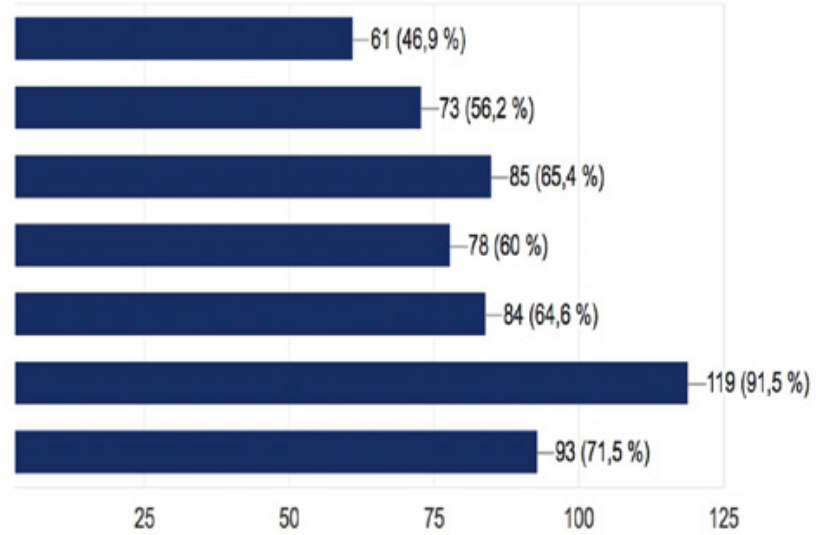

Figura 3. Principales ventajas que los estudiantes de pregrado de Medicina Interna I, II y III encuentran en las actividades presenciales.

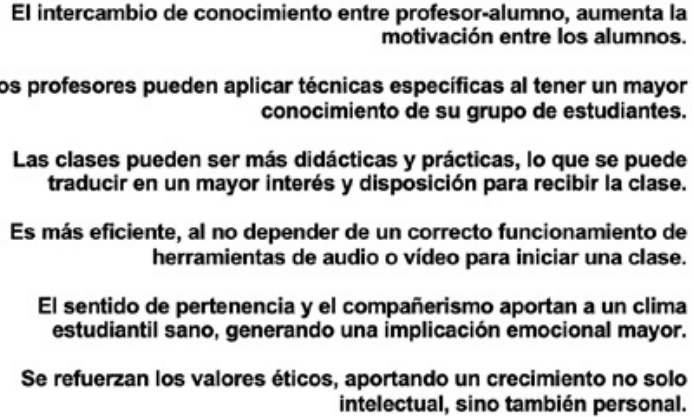

crecimiento intelectual, personal y emocional, mejorando el sentido de pertenencia, el compañerismo y el clima estudiantil.

En cuanto a las desventajas de la formación presencial (Figura 4), el 70\% consideran que los grupos numerosos generan distracciones, y el $65 \%$ observa que el tiempo de desplazamiento y factores ajenos generan mayor inversión en tiempo y dinero. E1 51\% piensa que las eventualidades presentadas por anormalidades académicas afectan el plan de estudios, y el $45 \%$ cree que la rigidez en el cumplimiento de horarios puede afectar la buena disponibilidad del estudiante para recibir información.

El 28\% de los estudiantes evalúan su compromiso con las actividades virtuales como muy bueno, para el 51\% es bueno. El 18\% lo consideró regular, el 2.3\% malo, y el $0.7 \%$ pésimo. El compromiso de la universidad con las actividades virtuales fue evaluado por el $42 \%$ como muy bueno, por el $47.2 \%$ como bueno, por el $8.5 \%$ como regular; el $1.5 \%$ lo consideró malo, y el $0.8 \%$, pésimo. Con

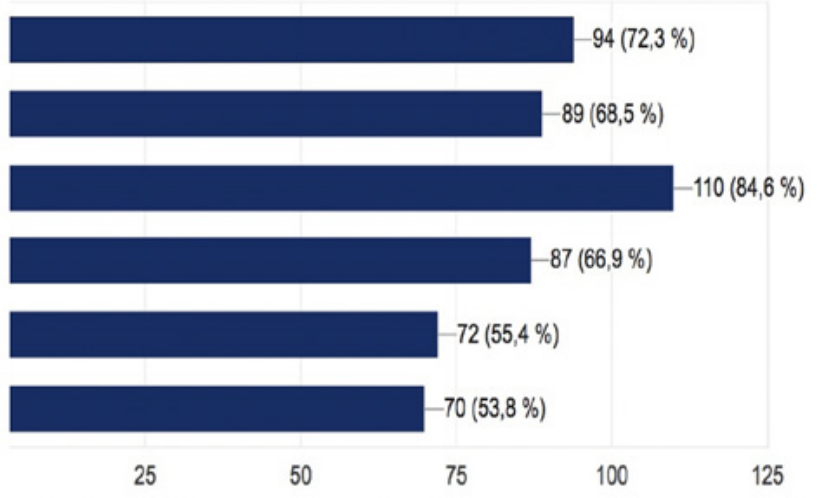

respecto a los docentes, el compromiso en las actividades virtuales fue calificado por el $36 \%$ como muy bueno, por el 54\% como bueno, por el $9.2 \%$ como regular y el $0.8 \%$ lo calificó como malo. Cuando se analiza, según el semestre de formación, en V semestre, el 21\% lo valoró como muy bueno, el 63\% bueno y el 16\% regular; en VI semestre, el $40 \%$ lo consideró muy bueno, el $48 \%$ bueno, el 8\% regular, y el 2\% malo; en VII semestre, el 50\% lo estimó muy bueno, el $48 \%$ bueno y el $2 \%$ regular.

Solo teniendo en cuenta esta pandemia, el 92\% de los estudiantes ponderó que la enseñanza virtual recibida fue adecuada. E1 66\% de los estudiantes calificó como buena la calidad de la enseñanza virtual brindada, el $32 \%$ regular y el $2 \%$ mala. Un $9 \%$ evaluó las actividades virtuales como mejores que las presenciales, el $43 \%$ las consideró iguales y el $48 \%$ peores $(62 \%$ en $\mathrm{V}, 48 \%$ en VI y $30 \%$ en VII). Un $59 \%$ consideraría útil continuar realizando algunas actividades de manera virtual (44\% en V, 60\% en VI y 80\% VII). 
Figura 4. Principales desventajas que los estudiantes de pregrado de Medicina Interna I, II y III encuentran en las actividades presenciales.

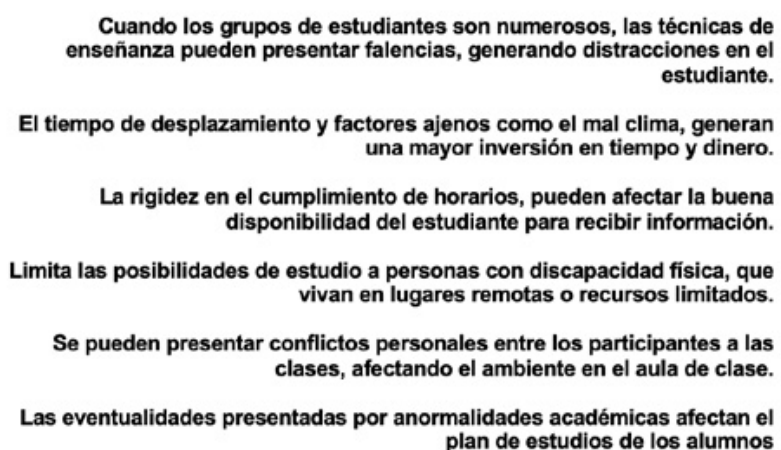

\section{Consideraciones}

En los últimos años, muchas escuelas de medicina han adaptado tecnologías y modelos para proporcionar experiencias de aprendizaje virtual; sin embargo, pocas actividades de la Facultad de Medicina de la UPB se habían implementado de esta manera, a pesar de la disponibilidad de plataformas. Esta pandemia nos sorprendió y fue necesario transformar súbitamente los cursos y actividades a la virtualidad. La transición de entorno de la facultad al del hogar tiene como resultado el aislamiento y mayor uso de herramientas y aplicaciones tecnológicas, así como dificultades para establecer límites entre el trabajo y el hogar, lo que afecta al profesorado, los estudiantes y el personal de apoyo.

Muchos docentes deben continuar su actividad asistencial, lo que implica no disponer del tiempo extra y tener disponibilidad o voluntad para implementar y desarrollar actividades virtuales.

La formación de la identidad profesional se basa en la enseñanza y en el modelamiento del rol en el entorno clínico, con el alumno como un aprendiz bajo supervisión. Por lo tanto, lo ideal es que el estudiante tenga relación directa con pacientes, docentes y entornos sanitarios en el "mundo real", donde se pone en práctica y fortalece sus bases teóricas. Pero, es innegable que, con la aparición de esta pandemia altamente contagiosa, el riesgo de que los estudiantes contraigan o transmitan el virus sin saberlo, sumado a la falta de pruebas de COVID-19 y equipos de protección personal (EPP) adecuados, a la cancelación de procedimientos quirúrgicos y citas y a las restricciones de centros de práctica, es imposible la realización de las prácticas clínicas como habitualmente se realizaban ${ }^{4}$. Ante este panorama, se puede "manifestar preocupación", realizar quejas y generar controversia o buscar opciones que minimicen la dificultad "semitransitoria".



Según la presente evaluación, como era de esperar, los estudiantes prefieren las actividades presenciales. El contacto con los pacientes, los compañeros, los profesores y los ambientes asistenciales (hospitalarios y ambulatorios) son el eje de la formación médica. Sin embargo, más del $90 \%$ consideraron adecuada la enseñanza virtual recibida, así como el trabajo de adaptación de la universidad y sus docentes. E1 66\% calificó como buena la calidad de la enseñanza, un 52\% la consideró igual o mejor que la presencial, y un 59\% consideraría útil continuar con algunas actividades virtuales. Menos del 20\% de los estudiantes opinó que hubiera sido mejor cancelar el semestre.

Es claro que los encuestados consideran que se aprende menos en las sesiones virtuales; pero, reconocen ciertas ventajas de la educación virtual, principalmente por el acceso permanente a los materiales, el mejor aprovechamiento del tiempo y el menor gasto de dinero. Con respecto a las desventajas manifestadas, algunas podrían ser optimizadas, principalmente la referente a la conexión a Internet (91\%). También es posible brindar asesoría para mejorar el uso del tiempo (71\%), la disciplina (65\%), las técnicas de aprendizaje (56\%) y los métodos de estudio (46\%).

Llama la atención que la experiencia con las actividades virtuales tuvo menor aprobación por los estudiantes de niveles inferiores. $\mathrm{E} 170 \%$ de los estudiantes de $\mathrm{V}$ semestre considera que se aprende menos en una sesión virtual que en una presencial, versus el $42 \%$ en VII semestre. Solo el 21\% de los estudiantes de V consideró el compromiso de sus docentes como muy bueno, versus un $40 \%$ en VI, y un 50\% en VII. Las actividades virtuales fueron consideradas como peores que las presenciales por un $62 \%$ de los encuestados en V, 48\% en VI y 30\% en VII. Considerarían útil continuar realizando algunas actividades de manera virtual el 44\% de los estudiantes en V,el 60\% en VI y el 80\% en VII. Debe entenderse que, 
durante el V semestre, se da la primera aproximación a la formación clínica.

Los datos sobre la experiencia en educación médica durante la pandemia aún son escasos. Se reportó la percepción de estas actividades en 77 estudiantes de pediatría (pregrado $13 \%$ y postgrado $87 \%$ ) en la India. E1 $97 \%$ consideró que las sesiones eran relevantes para sus necesidades de aprendizaje y práctica clínica. La mayoría percibe que las sesiones se adaptan a su nivel (99\%) y que las sesiones son interesantes y agradables (95\%). Todos consideraron que las clases en línea deberían formar parte del plan de estudios. Algunos respondieron que estas sesiones en línea rompían la rutina monótona, eran una buena utilización del tiempo y el material era de fácil acceso. Se sintieron motivados a leer los temas y el trabajo les ayudó a no pensar en COVID-19 y a dormir tranquilos. Los factores que con más frecuencia obstaculizaban el aprendizaje fueron la limitación del número de participantes, la limitación del tiempo de las sesiones y las fallas técnicas durante estos encuentros educativos virtuales. Estas deficiencias se intervinieron posteriormente mediante la compra de una versión avanzada del programa informático que permitió disponer de más tiempo y mejores oportunidades para interactuar ${ }^{5}$.

Con el creciente uso de la tecnología en la educación, el aprendizaje en línea se ha convertido en un método de enseñanza común. En 2019 se publicó un metanálisis con el objetivo de evaluar si el aprendizaje en línea, comparado con el presencial, mejora la aprehensión de los conocimientos en los estudiantes de medicina de pregrado. Los estudios fueron incluidos de acuerdo con los siguientes criterios: participantes (estudiantes universitarios de medicina), intervenciones (aprendizaje en línea, incluido el electrónico, por medios electrónicos, cursos masivos abiertos en línea y a distancia por vídeo), comparaciones (enseñanza presencial en un aula, seminarios, observación conjunta de videoconferencias en el aula y lectura de documentos basados en textos o libros únicamente), resultados (conocimientos y aptitudes medidos por instrumentos de evaluación objetivos) ${ }^{6}$.

En el estudio se disponía de la puntuación media y la desviación estándar (DE) de la evaluaciones previas y posteriores a la prueba, o de las pruebas de retención para los grupos experimentales y de control. Se encontró una diferencia significativa entre el aprendizaje en línea y presencial para el conocimiento y habilidades, basados en las puntuaciones de las pruebas posteriores ( $\mathrm{DE} 0,81$; IC del 95\%: 0,43, 1,20; $p<0,0001 ; n=15)$. La única comparación basada en las puntuaciones de las pruebas de retención también fue significativa (DE 4,64; IC del 95\%: 3,19, 6,09; $p<0,00001)^{6}$.

Aunque no todos los estudios incluidos en el metanálisis informaron que el uso de métodos de aprendizaje en línea fuera más eficaz que el proceso presencial, ninguno concluyó que el estudio en línea fuera menos eficaz que los métodos presenciales, independientemente del método estadístico utilizado. No hay clara evidencia de que el aprender presencial funcione mejor. A pesar de algunas preguntas pendientes, los hallazgos del trabajo en cuestión ofrecen pruebas sobre la efectividad del aprendizaje en línea en el pregrado de medicina ${ }^{6}$. Aunque se necesitan más investigaciones para aclarar los efectos del E-Learning y las condiciones en que puede utilizarse eficazmente, se podría considerar como un método potencial en la enseñanza de la medicina en la universidad.

Una revisión del grupo Cochrane evaluó los efectos de los programas de enseñanza virtual comparados con enseñanza tradicional en profesionales de la salud (fueron excluidos estudiantes de pregrado). Se incluyeron 16 ensayos aleatorizados con 5679 profesionales. No hubo evidencia a favor o en contra de la formación virtual. Debido a la escasez de datos, no fue posible profundizar en diferentes subgrupos ${ }^{7}$.

Según nuestra encuesta, es prudente evaluar, verificar y facilitar previamente el acceso a Internet por parte de los estudiantes (uno de cada cinco manifestó tener dificultades con el acceso, y nueve de cada 10 lo consideran la principal desventaja). Es necesario concientizar a la comunidad académica, principalmente a algunos docentes, de la gravedad y posible duración de la pandemia, y los profundos cambios que puede ocasionar en la formación actual y futura de los estudiantes de medicina, para adaptar su actitud y los cursos, contenidos y metodologías a las opciones de virtualidad disponibles. Deben evaluarse en todos los niveles, pero, principalmente, en los iniciales, las dificultades que se presentaron, que explicarían la percepción desfavorable de la actividad virtual, pues desafortunada o afortunadamente esta modalidad va a permanecer durante el futuro inmediato, $y$ es posible que deba adaptarse posteriormente para muchas actividades, para lo que hay que considerar sus ventajas y desventajas.

Recientemente se han identificado algunas barreras y soluciones entre los educadores médicos para la implementación del aprendizaje en línea en la educación médica, que se pueden utilizar para mejorar las prácticas institucionales y de los educadores en línea. Dentro de las barreras detectadas y sus posibles soluciones están ${ }^{8}$ :

\section{Déficit de habilidades}

La falta de conocimientos, en particular técnicos, es una de las barreras con que tropezaban los educadores cuando se ocupaban del desarrollo y la aplicación de la enseñanza en línea. La insuficiencia de conocimientos de informática, junto con una infraestructura deficiente, inhiben la voluntad o la capacidad de los educadores de participar en el desarrollo o la prestación de servicios de aprendizaje en línea. 
Se propone como solución lo siguiente: compromiso, para obtener los conocimientos necesarios. La participación en el aprendizaje electrónico, incluido el desarrollo de esos programas, es importante para obtener conocimientos para la práctica de la enseñanza. Es importante proporcionarle al profesorado las habilidades necesarias, a través de la formación sobre el uso de las diferentes herramientas. Para asegurar el éxito es crucial que el profesorado reciba la mencionada formación, estas capacitaciones deberían ser obligatorias para la continuidad y contratación de los docentes.

\section{Tiempo}

Los educadores médicos ya están bajo presión para encontrar tiempo para gestionar la enseñanza, la investigación y mantener un equilibrio entre la vida laboral y los compromisos de la vida personal. En este contexto, la falta de tiempo para dedicar al dominio, desarrollo y aplicación de las herramientas de aprendizaje en línea puede considerarse una barrera. Esta expectativa de tiempo a invertir puede considerarse perjudicial para el sistema pedagógico del propio educador, junto con las nociones preconcebidas de que las herramientas informáticas "siempre tardan más de lo esperado".

Curiosamente, la falta de tiempo parece vinculada a la falta de incentivos para participar en el aprendizaje en línea o electrónico. Es fundamental que los educadores dispongan del tiempo para familiarizarse y participar en el uso de este tipo de herramientas. La adopción de herramientas digitales puede, de hecho, liberar tiempo que les permita a los educadores médicos aprender conceptos y reflexionar sobre las prácticas.

\section{Infraestructura}

En muchos casos, la falta de infraestructura y tecnología (mala calidad de los servicios), como el acceso intermitente a Internet, el acceso limitado a las computadoras y una infraestructura física deficiente, pueden ser un obstáculo. Se podría considerar el costo de establecer la infraestructura correcta no como una barrera sino como una posible solución a una barrera.

\section{Falta de comunicación}

La adopción y aplicación del aprendizaje electrónico se considera a menudo un proceso que se adopta de manera polarizada, teniendo lugar solo en algunos departamentos de una institución, con falta de comunicación interdepartamental que se considera un obstáculo, lo que genera una percepción de dificultad para el intercambio activo de ideas y conocimientos.
Una de las formas más importantes en que la aplicación de los programas de aprendizaje en línea puede completarse con éxito es incluir a todos los interesados y departamentos pertinentes, con la adopción de nuevos enfoques para facilitar la colaboración. Se destaca la necesidad de que los administradores del aprendizaje electrónico establezcan mecanismos adecuados para disponer de recursos humanos cualificados y dedicados, asignar recursos financieros y prestar apoyo a todas las partes interesadas en función de sus necesidades. Es imperativo prestarle apoyo institucional al profesorado, para que pueda seguir participando en los programas en línea. Se requiere una estrategia institucional que facilite la aplicación de las competencias clave y la adopción de metodologías por parte del profesorado al poner en práctica el aprendizaje en esta modalidad.

\section{Actitud}

Finalmente, la actitud negativa de los educadores a la hora de comprometerse con las nuevas tecnologías y herramientas es una barrera para el desarrollo y aplicación del aprendizaje digital. Los educadores se sienten abrumados con el proceso de involucrarse con las nuevas herramientas y tienen poca paciencia para apropiar cuestiones técnicas. Esa sensación, derivada de un conocimiento limitado de una herramienta o de una formación inadecuada respecto a ella. Dicha sensación puede estar influyendo en la actitud de algunos educadores cuando se les pide que se comprometan a aplicar y desarrollar prácticas de aprendizaje en línea. Por consiguiente, fomentar un cambio de normas y actitudes es una solución importante para la introducción y la aplicación del aprendizaje online en la enseñanza de la medicina. La adopción de estos nuevos instrumentos puede, de hecho, producir una experiencia positiva e, incluso, romper las nociones preconcebidas.

En la UPB, las actividades presenciales y prácticas fueron suspendidas en su totalidad, por lo que no fue posible evaluar la experiencia durante este período, pero, es claro que deben retomarse gradualmente en el futuro cercano. Hay incertidumbre sobre cuánto tiempo persistirá esta situación y un reconocimiento cada vez mayor de que puede haber períodos en el futuro, después de la reincorporación a un entorno "nuevo normal", en los que tal vez se requieran nuevamente cuarentenas y distanciamiento social.

El reto consiste en proporcionar experiencias auténticas de pacientes a los estudiantes de medicina como un componente clave de la educación. ¿Qué podrían hacer los educadores para crear experiencias para los estudiantes que suelen ser asignados a rotaciones de pacientes hospitalizados o ambulatorios? Las opciones están en 
continua evolución, pero pueden incluir la consolidación y el desplazamiento de las sesiones didácticas clínicas en línea más temprano para permitir la entrada posterior en el entorno clínico; la creación y el uso de casos virtuales disponibles; la modificación del calendario académico para intercambiar experiencias posteriores (por ejemplo, trabajos académicos) y aplazar las rotaciones clínicas; la participación de los estudiantes en el entorno de la telemedicina, incluidas las asignaturas optativas basadas en las experiencias que los estudiantes persiguen para que puedan asistir y aprender en esta situación crítica.

Algunas escuelas sugieren eliminar las practicas optativas o "menos importantes" para reemplazarlas por las actividades que se vieron afectadas por la pausa ${ }^{1,4}$. Algunas escuelas de medicina, como la de la Universidad de Washington, han publicado su estrategia para enfrentar la pandemia con los estudiantes de cirugía9

La epidemia de COVID-19 puede implicar una transformación duradera en la medicina y, en general, en la vida. Ha sido considerada por algunos como un "cisne negro". En medio de esta crisis, es crucial que la comunidad educativa aprenda de la experiencia y de prioridad a un enfoque progresista y académico a medida que se implementan soluciones prácticas. Por consiguiente, es importante que las escuelas de medicina y sus educadores sean conscientes de los obstáculos y soluciones para el desarrollo y aplicación del aprendizaje virtual y de la necesidad de que exista promoción y apoyo al uso de esta metodología, para asegurar que este modo de enseñanza se mantenga y actualice para reflejar la naturaleza dinámica de la tecnología y la educación médica.

De esta manera, los educadores y estudiantes de medicina estarán mejor preparados para los desafíos que se plantean. El aprendizaje en línea tiene consecuencias para los educadores que optan por enseñar mediante esta modalidad, entre ellas, una mayor responsabilidad, la necesidad de modificar el estilo de enseñanza y de mantener una comunicación continua y significativa.

Aún se desconoce cuán efectivo es el aprendizaje línea través de la Red para la educación médica de pregrado, pero es necesario reconocer que este tipo de formación tiene sus propias ventajas para mejorar el aprendizaje de los estudiantes y debe considerarse un posible método de enseñanza. Para garantizar la eficacia de la educación virtual, se deben evaluar rigurosamente los principios de diseño de los materiales didácticos digitales, los objetivos de aprendizaje, los resultados y las preferencias y características de los estudiantes.

Este no es solo un momento para contribuir al avance de la educación médica en el marco de la innovación y transformación curricular activa, sino que puede ser un momento fundamental para muchas disciplinas de la medicina. La reflexión y la evaluación deben seguir, se propone continuar evaluando la experiencia de los diferentes cursos y niveles con las metodologías implementadas, así como la proposición de diferentes formas de acercar y adaptar a la comunidad académica con estas modalidades.

\section{DECLARACIÓN DE CONFLICTO DE INTERESES}

El autor declara no tener ningún conflicto de intereses.

\section{REFERENCIAS}

1. Rose S. Medical student education in the time of COVID-19. JAMA (en prensa). 2020;10.1001/ jama.2020.5227.

2. Buja, LM. Medical education today: All that glitters is not gold. BMC Med Educ 2019; 19:110.

3. Han E, Yeo S, Kim M, Lee Y, Park K, Roh H. Medical education trends for future physicians in the era of advanced technology and artificial intelligence: An integrative review. BMC Med Educ 2019; 19:460.

4. Ferrel MN, Ryan JJ. The impact of COVID-19 on medical education. Cureus. 2020; 12: e7492.

5. Agarwal S, Kaushik JS. Student's perception of online learning during COVID Pandemic. Indian J Pediatr. 2020; 87(7):554.

6. Pei L, Wu H. Does online learning work better than offline learning in undergraduate medical education? A systematic review and meta-analysis, Medical Education Online. 2019; 24:1

7. Vaona A, Banzi R, Kwag KH, Rigon G, Cereda D, Pecoraro V, et al. E-learning for health professionals. Cochrane Database Syst Rev. 2018; 1(1):CD011736.

8. O'Doherty D, Dromey M, Lougheed J, Hannigan A, Last J, McGrath D. Barriers and solutions to online learning in medical education -an integrative review. BMC Med Educ 2018; 18:130.

9. Calhoun KE, Yale LA, Whipple ME, Allen SM, Wood DE, Tatum RP. The impact of COVID-19 on medical student surgical education: Implementing extreme pandemic response measures in a widely distributed surgical clerkship experience. Am J Surg. 2020; S0002-9610(20)30229-4. 